\title{
Flow topology and acoustic emissions of trailing edge serrations at incidence
}

\author{
Carlos Arce León ${ }^{1,2}\left(\right.$ Daniele Ragni $^{1} \cdot$ Stefan Pröbsting $^{1} \cdot$ Fulvio Scarano $^{1}$ \\ Jesper Madsen ${ }^{2}$
}

Received: 6 October 2015 / Revised: 12 April 2016 / Accepted: 28 April 2016 / Published online: 11 May 2016

(C) The Author(s) 2016. This article is published with open access at Springerlink.com

\begin{abstract}
The flow past a NACA 0018 airfoil with sawtooth trailing edge serrations has been investigated using stereoscopic particle image velocimetry (PIV). The serration flap angle and airfoil incidence are varied in order to study the effect of secondary flow establishing between the suction and pressure sides of the serrations. The flow topology around the serrations is inferred from the analysis of time-averaged streamlines close to the airfoil surface and from the wall-normal flow velocity in between serrations. Additional PIV measurements with a plane in cross-flow highlight the formation of streamwise vortex pairs. The flow behavior is further characterized in terms of its turbulence statistics. Noise emissions are measured with an acoustic phased array in combination with beamforming. The serrations are found to be effective in reducing noise, and their application is studied for different degrees of airfoil incidence and serration flap angle.
\end{abstract}

\section{Introduction}

Broadband airfoil noise emissions that originate due to the interaction of the airfoil turbulent boundary layer with the sharp trailing edge (Brooks et al. 1989) have been shown to be effectively reduced using trailing edge serrations. Evidence of this has been observed in acoustic measurements

Carlos Arce León

C.A.ArceLeon@tudelft.nl

1 Department of Aerospace Engineering, Delft University of Technology, Kluyverweg 1, 2629 HS Delft, The Netherlands

2 Aerodynamics and Acoustics Group, LM Wind Power, Jupitervej 6, 6000 Kolding, Denmark performed both in wind tunnel experiments (Gruber et al. 2013; Moreau and Doolan 2013; Dassen et al. 1996) and on full-scale wind turbines (Schepers et al. 2007; Oerlemans et al. 2009). For the latter, airfoil self-noise reduction is relevant in relation to the observance of noise limits established by local regulations. Furthermore, experimental studies related to the flow around serrations and surface pressure characterization have also been previously performed (Chong and Vathylakis 2015; Finez et al. 2011; Arce et al. 2015).

An analytical model for the prediction of the noise emitted from a sawtooth serrated trailing edge has been proposed by Howe (1991a, b). It yields the nondimensional acoustic power spectrum, $\Psi$, of the serrated trailing edge with respect to the reference spectrum of the straight trailing edge, $\Psi_{0}$, as a function of serration wavelength, $\lambda$, and amplitude, $2 h$ (see Fig. 1 for the geometric definition of $\lambda$ and $2 h)$

$\Psi(\omega) \approx \frac{\Psi_{0}(\omega)}{1+(4 h / \lambda)^{2}}$,

where $\omega$ is the angular frequency of the acoustic pressure fluctuations.

The above relation has been derived under the assumption of frozen turbulence convected across the trailing edge. In particular, the time-average streamlines are assumed to remain aligned with the freestream direction. For an airfoil at incidence, the transverse fluid motion induced by the pressure unbalance between suction and pressure side limits applicability of the theory as commented by Howe (1991b).

Experimental studies (Gruber et al. 2013; Moreau and Doolan 2013) have reported less effective noise reduction for serrated trailing edges than that predicted with the above-mentioned model. The mismatch could be ascribed 


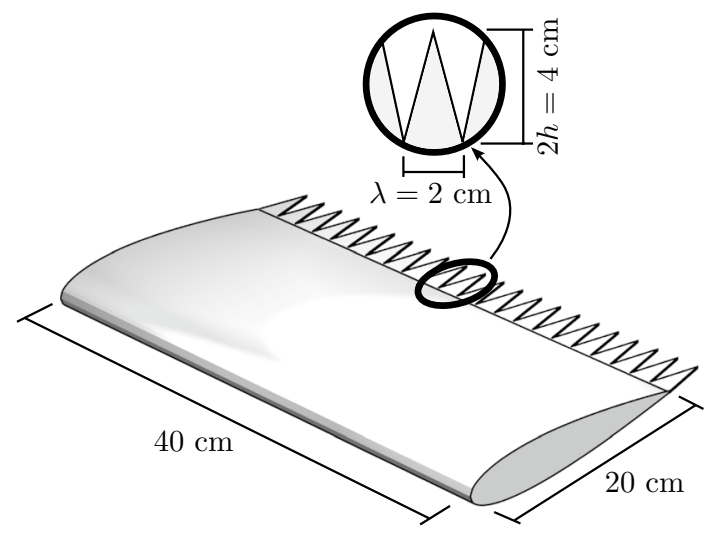

Fig. 1 Dimensions of the airfoil and serrations

to a number of flow parameters which the theory does not account for, and which may lead to a departure from the predicted behavior. A more thorough understanding of the flow field around the serrations is therefore needed to unambiguously identify potential reasons of the discrepancy between the above model and experimental observations.

While wind tunnel experiments have revealed that a reduction in noise is often measured when using serrations, a slight increase in noise at relatively higher frequencies has also been reported (Moreau et al. 2011; Gruber 2012). It has been argued in Dassen et al. (1996) and Oerlemans et al. (2009) that the noise increase at higher frequencies might be due to the misalignment of the serrations with the flow in the wake of the airfoil. Gruber et al. (2011) has ascribed this phenomenon to an increased turbulence activity between the serration teeth.

In order to relate the noise reduction efficiency to flow field effects, the present work combines stereoscopic particle image velocimetry (PIV) and acoustic phased array measurements of sawtooth trailing edge serrations on a NACA 0018 airfoil. The experiments are conducted comparing the serrated trailing edge with the straight edge airfoil. The pressure unbalance between the serration upper and lower surfaces is varied by placing the airfoil at incidence, and accentuated when the inclination of the serrations is also varied.

The time-averaged flow topology is first characterized to determine the streamline distortion. The analysis of the turbulent velocity fluctuations yields the properties of the boundary layer along the serrations. The noise emitted by the serrated trailing edge is monitored with a microphone array yielding the power spectral density of the acoustic pressure fluctuations in the relevant frequency range.

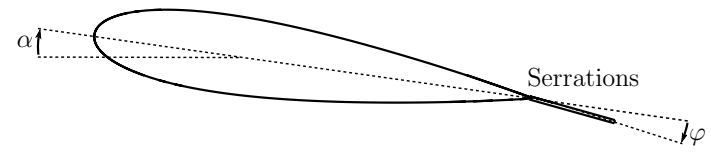

Fig. 2 Airfoil cross section and serrated trailing edge flap. Angle of attack, $\alpha$, and the serration flap angle, $\varphi$, are shown

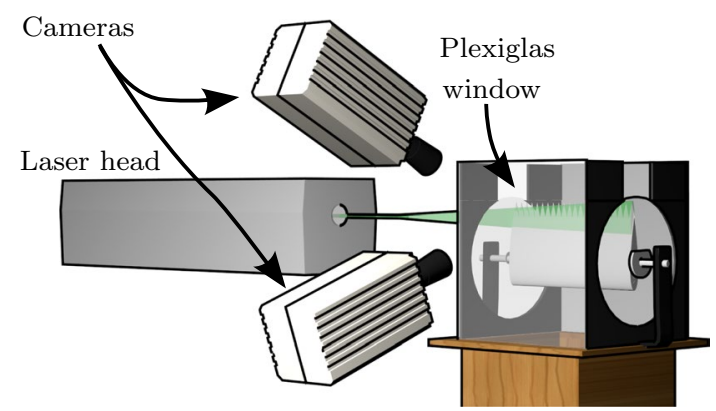

Fig. 3 Setup used in the near-wall (camera and laser position shown) and the cross-flow plane measurements

\section{Experimental setup}

Experiments were conducted in a low-speed wind tunnel with vertical exit (V-Tunnel) operated within the aerodynamics laboratories of the Aerospace Engineering Department at TU Delft. The contraction ratio of 60:1 produces a flow at the exit $\left(40 \times 40 \mathrm{~cm}^{2}\right)$ with turbulence intensity below $1 \%$. The operating velocity ranges from $10 \mathrm{~m} / \mathrm{s}$ to $45 \mathrm{~m} / \mathrm{s}$.

A NACA 0018 airfoil profile has been CNC-machined into an aluminum model with removable trailing edge inserts, a chord $C=20 \mathrm{~cm}$ and a span of $40 \mathrm{~cm}$. The length of the serrations is $2 h=4.0 \mathrm{~cm}$ with $2 h / \lambda=2$, such that $\lambda=2.0 \mathrm{~cm}$ is the serration wavelength (see Fig. 1). The serration panel thickness is $1.0 \mathrm{~mm}$. When measurements are taken in respect to the straight trailing edge, it is the original trailing edge of the NACA 0018 airfoil which is used.

The relevant parameters inducing an unbalance in the pressure between the serration upper and lower surfaces are the airfoil angle of attack, $\alpha_{g}$, and the serration flap angle, $\varphi$. Figure 2 shows the convention used, where a positive value of $\varphi$ indicates an inclination of the flap angle toward the airfoil pressure side.

The airfoil was placed at the exit of the wind tunnel and held by means of side plates. Three experimental configurations were needed to measure the flow at three different plane locations, namely the near-wall plane, cross-flow plane, and wall-normal plane. A sketch of the setup for the near-wall plane and the cross-flow plane experiments can 


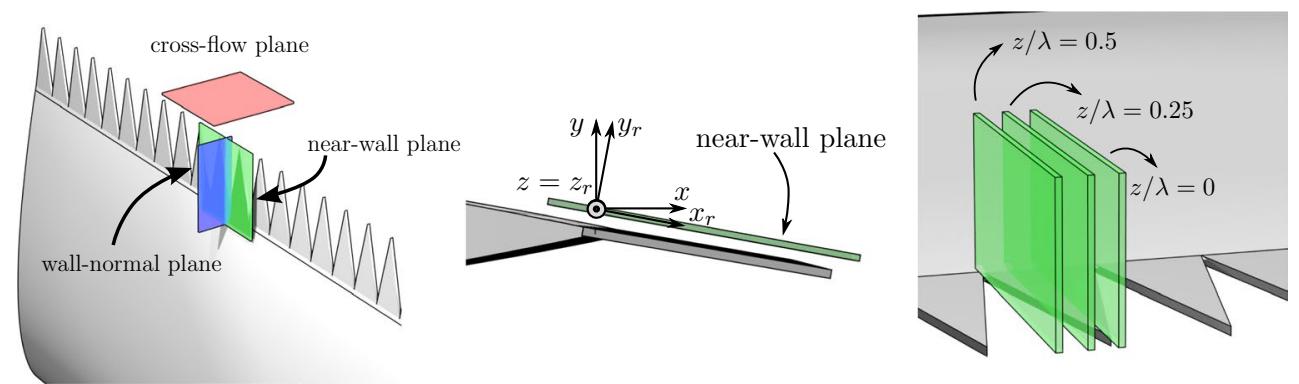

Fig. 4 Left PIV measurement planes along different direction. Only one of three wall-normal planes is indicated for clarity. Center coordinate system for the near-wall plane. Right position of wall-normal measurement planes

be seen in Fig. 3. The wall-normal plane experiment is shown in Fig. 4, right.

Boundary layer transition was forced by a tripping element at $x / C=0.175$. The trip is composed of randomly distributed roughness elements (carborundum with nominal grain size of $0.6 \mathrm{~mm}$ ) over a width of approximately $10 \mathrm{~mm}$. The onset of turbulence past the tripping element was verified with a stethoscope probe. For the baseline configuration $\left(\alpha=0^{\circ}\right)$, the boundary layer thickness at the airfoil trailing edge was measured with PIV to be $\delta_{99}=15.9 \mathrm{~mm}$. PIV experiments are conducted at freestream velocity $U_{\infty}=20 \mathrm{~m} / \mathrm{s}$, corresponding to a chord-based Reynolds number of $2.6 \times 10^{5}$.

\subsection{Stereoscopic PIV}

The coordinate system is defined with $x, y$, and $z$ along the freestream, vertical and spanwise directions, respectively. The origin is located at the trailing edge of the airfoil and at the serration tooth centerline. The velocity vector is represented by the components $u, v$, and $w$, in $x, y$, and $z$ coordinate directions, respectively.

Three-component velocity measurements were conducted using stereoscopic PIV. The air flow was seeded with $1 \mu \mathrm{m}$ tracer particles of evaporated water-glycol-based fog fluid. Illumination is obtained with a Quantronix Darwin Duo, Nd:YLF laser $(2 \times 25 \mathrm{~mJ}$ at $1 \mathrm{kHz})$ and laser optics is used to form a sheet of approximately $1.5 \mathrm{~mm}$ thickness.

The measurement configurations are illustrated in Fig. 4, left. The near-wall plane $(x-z, y=2 \mathrm{~mm})$ enables to quantify the streamlines distortion and to study the nearwall flow topology. The laser sheet is maintained parallel to the surface of the serrations (see Fig. 4, center), and thus, results are presented based on a coordinate system rotated about the $z$ axis $\left(x_{r}, y_{r}, z_{r}\right)$. The cross-flow plane $(y-z, x=60 \mathrm{~mm})$ inspects the streamwise vortices emanating from the serrated trailing edge. Finally, three wallnormal planes $(x-y, z=0, \lambda / 4, \lambda / 2)$ are chosen that span one serration element and determine the properties of the turbulent boundary layer upstream and spanwise across the trailing edge (see Fig. 4, right).

The imaging system is composed of two Photron Fastcam SA1 CMOS cameras $\left(1024 \times 1024 \mathrm{px}^{2}\right.$, pixel-pitch $20 \mu \mathrm{m} / \mathrm{px}, 5.4$ frames per second) equipped with Nikon NIKKOR macro-objective lenses and Scheimpflug adapters to correct for the misalignment between the object and sensor plane. With about $50 \mathrm{~cm}$ distance from the object and an angle of $40^{\circ}$ between the two cameras, the field of view covers an area of approximately $5 \times 5 \mathrm{~cm}^{2}$, resulting in a digital imaging resolution of $20 \mathrm{px} / \mathrm{mm}$.

Image pairs are acquired with a time separation of $50 \mu \mathrm{s}$ at a rate of $250 \mathrm{~Hz}$ for a total of 1500 instantaneous measurements $(6 \mathrm{~s})$. Image interrogation is performed with LaVision DaVis 8 software using a multipass stereo cross-correlation with a final window size of $16 \times 16 \mathrm{px}\left(0.8 \times 0.8 \mathrm{~mm}^{2}\right)$. An overlap factor of $75 \%$ between adjacent windows is applied, leading to a vector spacing of $0.2 \mathrm{~mm}$.

The typical measurement uncertainty is estimated by a linear propagation approach (Stern et al. 1999) and verified a posteriori using the statistical analysis method introduced by Wieneke (2015). The random and bias components are considered. Bias errors due to peak-locking (Westerweel 1997) are associated to the large imaging aperture ( $f$ / 5.6) yielding a diffraction spot of only half pixel diameter. This effect is mitigated by slightly defocusing the images (Raffel et al. 2007), bringing the particle image diameter to approximately $1.5 \mathrm{px}$. The verification is performed inspecting the histogram of particles image displacement, which in this case gives no evidence of peak-locking. The error due to finite spatial resolution depends on the characteristic length to be accurately measured in the PIV velocity fields. With a multi-pass cross-correlation algorithm with window deformation the amplitude of the fluctuation is measured with $<5 \%$ modulation when the window size is smaller than 0.6 times the length of the scale to be measured (Schrijer and Scarano 2008). Therefore, with $0.8 \mathrm{~mm}$ window size, flow structures down to $1.2 \mathrm{~mm}(0.13 \%$ of the boundary layer $)$ can be measured within $95 \%$ precision. Other systematic 


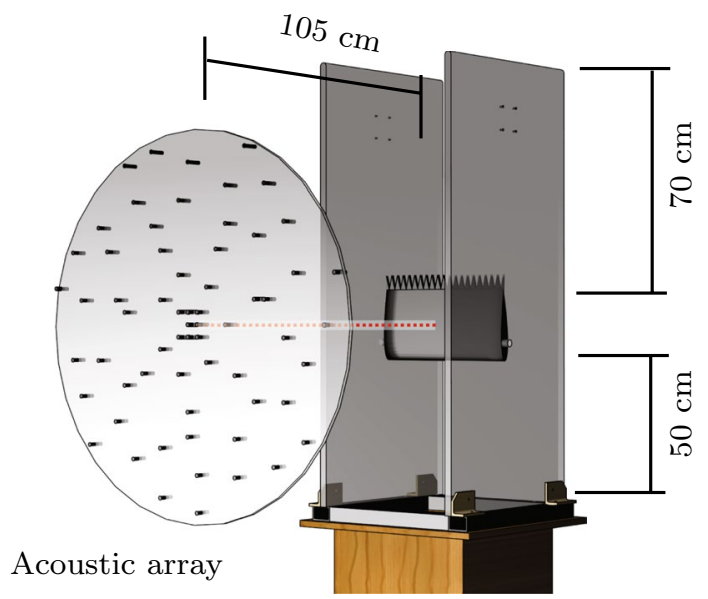

Fig. 5 Setup for the acoustic measurements (also used for the PIV measurements on the wall-normal plane)

sources of uncertainty, including calibration errors and lens distortion, are alleviated by iterative steps of a self-calibration procedure with a polynomial mapping of the images, via the $\mathrm{DaVis}$ software. A disparity vector smaller than $1 p x$ is reached for the stereo calibration (Raffel et al. 2007).

The random components of the uncertainty have been evaluated with a technique following the work of Wieneke (2015), where the degree of particle images matching is quantified with the analysis of the correlation peak width. Random errors vary along the measurement domain with $<1 \%$ and approximately $3 \%$ in the freestream and in the inner boundary layer regions, respectively. The uncertainty of the mean of the velocity, and the root mean square (RMS) of its fluctuations, also depends upon the size of the measured ensemble to form the statistics. In the present case, the uncertainty on the mean velocity is 0.01 and $2 \%$ on its RMS.

\subsection{Acoustic measurements}

The beamforming technique is applied by means of a phased microphone array. The airfoil is installed in the wind tunnel open test section, with side plates, with the airfoil leading edge located $50 \mathrm{~cm}$ downstream of the wind tunnel nozzle. The side plates end $70 \mathrm{~cm}$ downstream of the airfoil trailing edge. This allows sufficient spatial separation of the noise emanating from the trailing edge from other noise sources. A view of the experimental arrangement can be seen in Fig. 5, and a sample acoustic source map is shown in Fig. 6.

The microphone array consists of 64 electret-condenser PUI AUDIO 665-POM-2735P-R omni-directional microphones. The microphones have a sensitivity of $-35 \pm 2 \mathrm{~dB}$ (ref. $1 \mathrm{~V} / \mathrm{Pa}$ ) over a frequency range of $0.2-25 \mathrm{kHz}$. The array arrangement consists of a multi-arm logarithmic

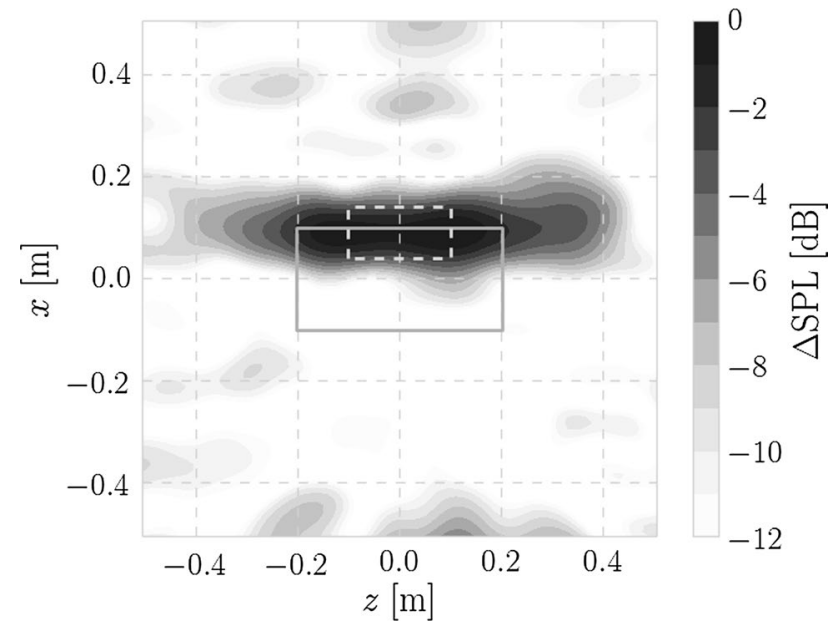

Fig. 6 Source map for serrated trailing edge with $\alpha_{g}=12^{\circ}$ and $\varphi=6^{\circ}, U_{\infty}=35 \mathrm{~m} / \mathrm{s}, f=4565 \mathrm{~Hz}$. The airfoil location (planform boundary) is indicated with the solid line, and the integration region with the dashed line

spiral configuration (Mueller 2002; Pröbsting et al. 2015b), designed for the suppression of side lobes below $-12 \mathrm{~dB}$ over the field of view, and frequency range between 1 and $5 \mathrm{kHz}$. The microphone array center is pointed straight toward the center of the airfoil and is located at a distance of $105 \mathrm{~cm}$.

Data acquisition is performed during $60 \mathrm{~s}$ at a sampling rate of $50 \mathrm{kHz}$. The sample block length used is 4096 , with an overlap of $50 \%$, resulting in a frequency resolution of $24.4 \mathrm{~Hz}$. A Hanning window is applied. The number of independent blocks in the ensemble average is $k=1465$, or $k=2930$ considering the $50 \%$ overlap. The normalized random error of the PSD estimate is given by $1 / \sqrt{k}$, which conservatively for $k=1465$ results in $\varepsilon_{\text {rand }} \approx 2.6 \%$, yielding a $95 \%$ confidence interval of $\approx 2 \varepsilon_{\text {rand }}= \pm 5 \%$.

A beamforming technique is applied following Sijtsma (2010). The resulting source power distribution is integrated over a region around the trailing edge to obtain the source power. This integration region was centered on the trailing edge and is $20 \mathrm{~cm}$ long in the spanwise direction, while it scales with the frequency-dependent beamwidth in the chordwise direction.

\subsection{Angle of attack correction}

In the acoustic measurements an open test section is necessary and was also used for the wall-normal plane PIV measurements. An indication of the effective angle of attack follows from Brooks et al. (1984), where the effective angle of attack $\alpha$ is corrected from the geometric one, $\alpha_{g}$ :

$\alpha=\alpha_{g} \frac{1+2 \sigma}{(1+2 \sigma)^{2}+\sqrt{12 \sigma}}$, 
Fig. 7 Cross-flow measurements showing streamwise vorticity for different permutations of $\alpha_{g}$ and $\varphi$ and $U_{\infty}=20 \mathrm{~m} / \mathrm{s}$. Mean flow direction in $v$ and $w$ is indicated with arrows in the four bottom right frames

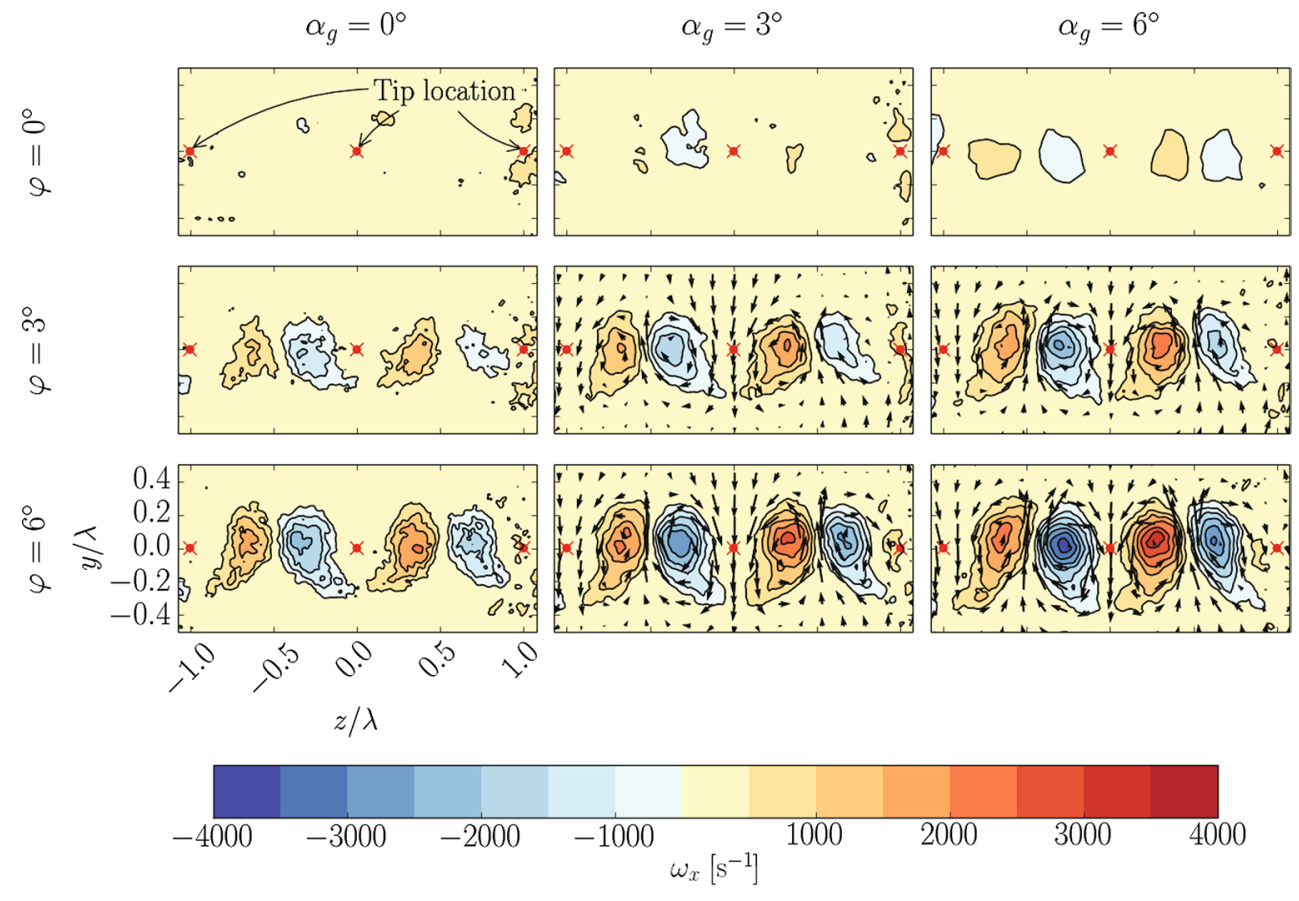

where $\sigma=\pi^{2} C^{2} / 48 H^{2}$. Here $C$ is the airfoil chord and $H$ is the wind tunnel nozzle height. Applying the corrections on the geometric values used in the experiments gives: $\alpha_{g}=12.0^{\circ} \rightarrow \alpha=6.6^{\circ}$, and $\alpha_{g}=6.0^{\circ} \rightarrow \alpha=3.3^{\circ}$. The remainder of the discussion will refer to $\alpha_{g}$. For the measurements in the cross-flow plane and in the near-wall plane (closed test section) it is assumed that $\alpha \approx \alpha_{g}$.

\section{Results and discussion}

\subsection{Mean flow topology}

Results of the cross-flow plane measurements are presented in Fig. 7, where the vorticity component, $\omega_{x}$, is shown for different configurations of flap angle and airfoil incidence. The position of the serration tip is projected on the measurement plane.

The presence of streamwise, counter rotating vortex pairs, emanating from the serrations is evident from these results. It is also shown that the circulation pertaining to these vortices is also more strongly correlated with the serrations flap angle, rather than with the incidence of the whole airfoil. An increase in $\varphi$ by $3^{\circ}$ for $\alpha_{g}=0^{\circ}$, increases the vorticity maximum by over $1000 \mathrm{~s}^{-1}$. Incrementing $\alpha$ by $3^{\circ}$, keeping $\varphi$ constant at $0^{\circ}$, increases the vorticity by just half the amount.

The vortex pairs produce a vigorous upwash motion in between the serrations and a downwash directly behind the serration tips, resulting from the pressure difference established between the two sides of the serrations once they are placed at an angle with respect to the airfoil chord. Similar flow behavior, with a transverse motion between the teeth toward the suction side, has been reported by Arina et al. (2012).

The streamwise vortices give an indication of secondary motions in the wake, in turn associated with the streamline distortion close to the surface of the trailing edge. These observations suggest consequently that the angle by which turbulent fluctuations are advected across the edge will depart from that of the serrations (defined below as $\theta_{0}$ ).

The result for the normalized wall-normal flow component, $v_{r} / U_{\infty}$, for the case where $\alpha_{g}=6^{\circ}$ and $\varphi=6^{\circ}$, can be seen in Fig. 8. The measurement of the straight trailing edge (located at $x=0$ ) is also shown for comparison. The outline of the serrated trailing edge is indicated.

On the pressure side (Fig. 8, middle), the flow is directed into the measurement plane over the entirety of the domain. This indicates that flow passes through it with regions of higher velocity in between adjacent serrations at around half a serration length. The velocity on the suction side (Fig. 8, right) exhibits two streamwise elongated regions with positive wall-normal velocity. These regions are consistent with the formation of streamwise vortices observed in the wake.

The near surface flow distortion is better observed in the measurement of $w_{r} / U_{\infty}$ (Fig. 9). The streamlines are affected to a different extent when airfoil angle of attack or serration flap angle is increased. The $w_{r}$ component of the streamlines has been scaled by a factor of four to clarify 
Fig. 8 Color contours of timeaveraged wall-normal velocity distribution for $\alpha_{g}=6^{\circ}$ and $\varphi=6^{\circ}$. Straight trailing edge (left), serrated pressure side (middle), and serrated suction side (right)

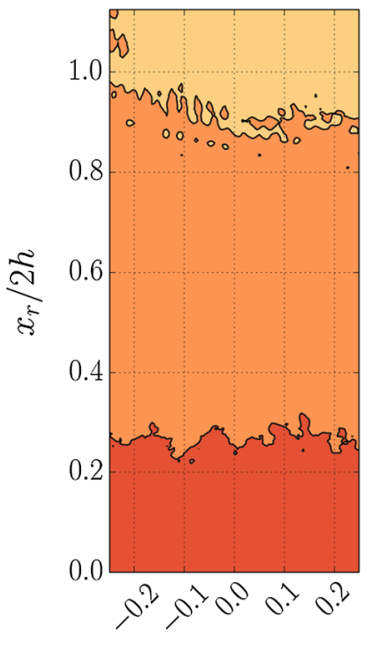

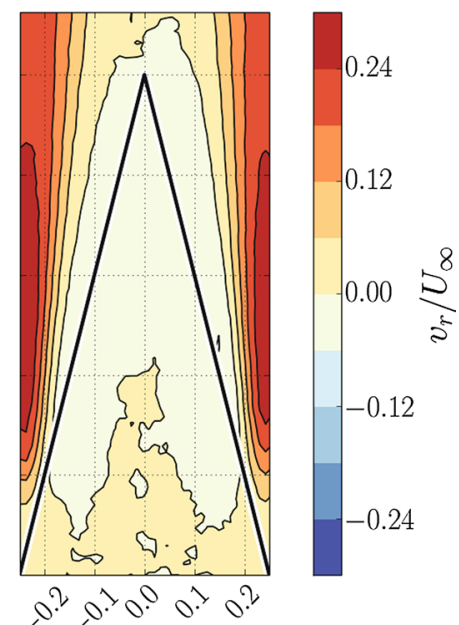
$z_{r} / 2 h$
Fig. 9 Near surface spanwise velocity (color contours) and a qualitative streamline pattern for varying $\alpha_{g}$ and $\varphi$. Pressure (top) and suction side (bottom)
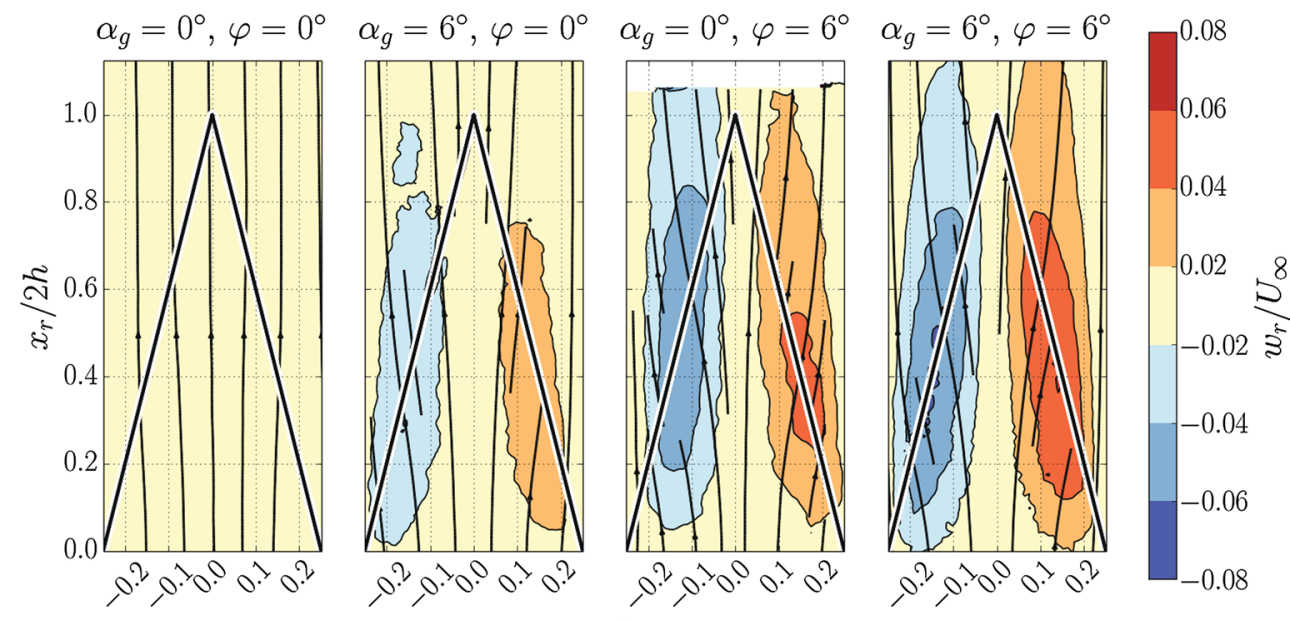
$z_{r} / 2 h$
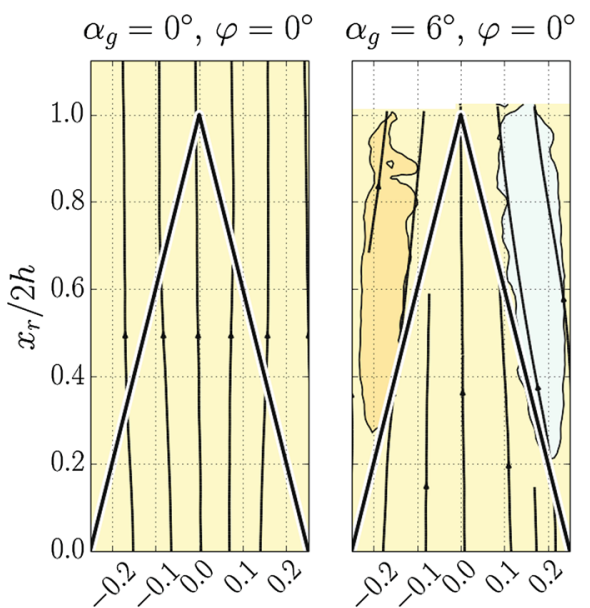

$\alpha_{g}=0^{\circ}, \varphi=6^{\circ}$
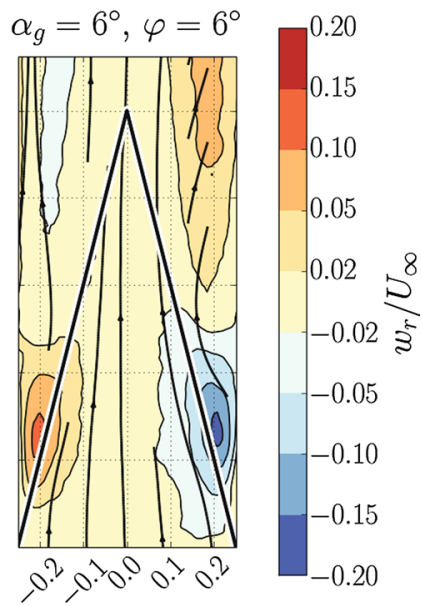

the direction to which the streamlines point. The effect of flap angle is again more pronounced than that of the airfoil angle of attack.
The near surface flow at the pressure side (Fig. 9, top) exhibits deflection away from the serration centerline, $z_{r} / 2 h=0$. Instead, the streamlines on the suction side 
(Fig. 9, bottom) appear to converge toward the tip of the serration tooth. This result indicates that the serration trailing edge angle apparent to the mean flow streamlines is being modified by the secondary flow pattern established as a result of a pressure difference. The most direct implication is a discrepancy to the prediction obtained from the model in Howe (1991b).

In the analytical formulation from Howe (1991b), it is argued that flow properties around a distance $\delta$ from the serration edge are relevant to its noise reduction mechanism. When no airfoil incidence or serration flap angle is applied, the mean flow behavior near the serrations has been shown in Fig. 9 to adhere closely to the above assumption. The most notable modification due to serrations remains the change in the effective angle at which the turbulent flow structures convect over the trailing edge. The latter are responsible for noise generation, with the most effective condition for noise production given by streamlines being orthogonal to the edge.

On the suction side the streamlines tend to align even more with the edge, while on the pressure side the behavior is opposite and the streamlines tend to become normal to the edge. Even under these conditions, the serrations have been shown in multiple studies (Gruber 2012; Moreau et al. 2011) to be effective at reducing noise. This further suggests that, although the main working principle hypothesized by Howe (1991b) (to change the effective angle at which the noise generating flow structures convect over the trailing edge) remains true, the validity of frozen flow properties does not hold true.

The angle formed by the streamlines and the trailing edge plays a primary role in Howe's model [Eq. (1)]. Therefore, understanding how airfoil incidence or serration flap angle modify the flow behavior and the noise reduction is the relevant question addressed. The actual streamline topology obtained from the PIV analysis explains part of this phenomenon.

The angle at which the local streamline passes over the serration trailing edge will be referred to as $\theta$ and is illustrated in Fig. 10. Here $\theta_{0}$ represents the angle between the undisturbed streamline and the serration trailing edge, and $\gamma$ is the local flow deflection over the trailing edge, such that $\theta=\theta_{0}+\gamma$. The angle $\gamma$ is positive by a deflection that increases the total angle $\theta$.

The serration geometric parameters in Eq. (1) can be rewritten such that it becomes a function of the trailing edge angle. Since

$\cot \theta_{0}=4 h / \lambda$,

then, from Eq. (1),

$\Psi \approx \frac{\Psi_{0}}{1+\cot ^{2} \theta_{0}}=\Psi_{0} \sin ^{2} \theta_{0}$.

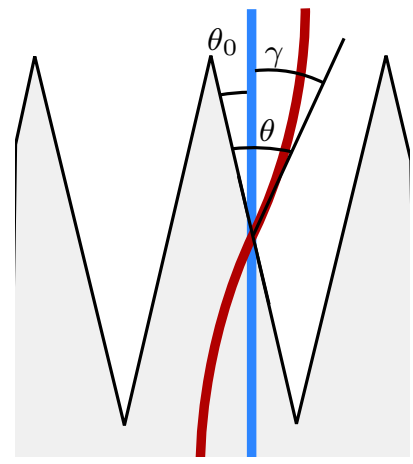

Fig. 10 Definition of the flow curvature angle, $\theta$, with respect to the serration trailing edge. Schematic situation on the airfoil pressure side

Correcting for the streamline deflection yields $\Psi \approx \Psi_{0} \sin ^{2}\left(\theta_{0}+\gamma\right)$.

The maximum observed value of $\gamma$ is charted in Fig. 11 for the pressure side. For $\varphi=6^{\circ}, \gamma$ approaches $4^{\circ}$. Figure 11 shows the difference in decibels between the predicted noise of the serrated trailing edge and that of a straight trailing edge. When the value of $\gamma$ increases, the serration is expected to become less effective in reducing the trailing edge noise. For the cases studied here, the serrations are expected to lose approximately $2 \mathrm{~dB}$ in noise reduction performance at the highest observed deflection only on the pressure side. A simplifying hypothesis is made here that the boundary layer properties (thickness and shape factor) along with the flow turbulence are unaltered by the presence of the serrations at varying values of $\varphi$.

From the suction side, instead, following Howe's approach, one expects an additional reduction of noise emission since the streamline deflection tends to reduce $\theta$ even further, with the flow becoming more parallel to the trailing edge.

There is evidence (see Blake 2012; Bertagnolio et al. 2014) that the contribution of noise between the pressure and suction sides is not equal and the peak noise level is likely to be strongly driven by the boundary layer thickness, suggesting that for a straight trailing edge airfoil the suction side is the dominant source of noise. Based on the observations made regarding the beneficial direction in which the flow is deflected on the suction side, this leads to the conclusion that the observed streamline distortion would further increase the overall noise reduction. This is not observed, and serration misalignment has instead been linked to an increase in noise in the higher frequencies while maintaining similar levels of reduction in the lower and mid-frequency ranges (Gruber et al. 2011).

Admittedly, it must be retained in mind that the value of $\gamma$ is not constant over the serration trailing edge, which should be considered for a quantitative measurement of it. Considering a correction based on the maximum deflection 
Fig. 11 Values of $\gamma_{\max }$ for different cases measured over the serration trailing edge (left). Expected influence of $\gamma$ on Eq. (4) (right)
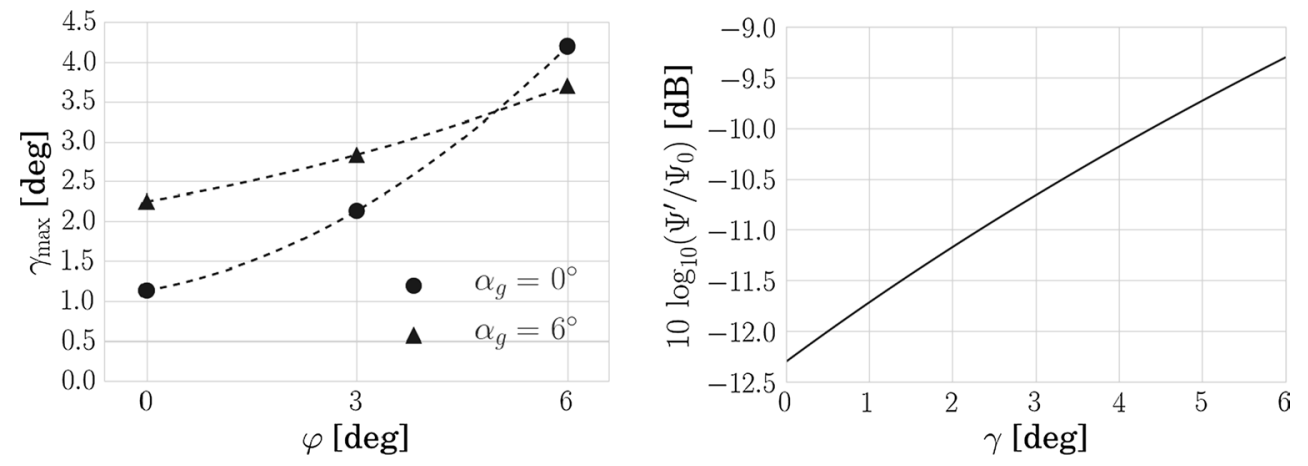

Fig. 12 Profiles over the wallnormal coordinate direction of $\overline{u_{r}}$ for the straight trailing edge and three spanwise locations of the serrated edge for $\alpha_{g}=0^{\circ}$ and $\varphi=0^{\circ}$

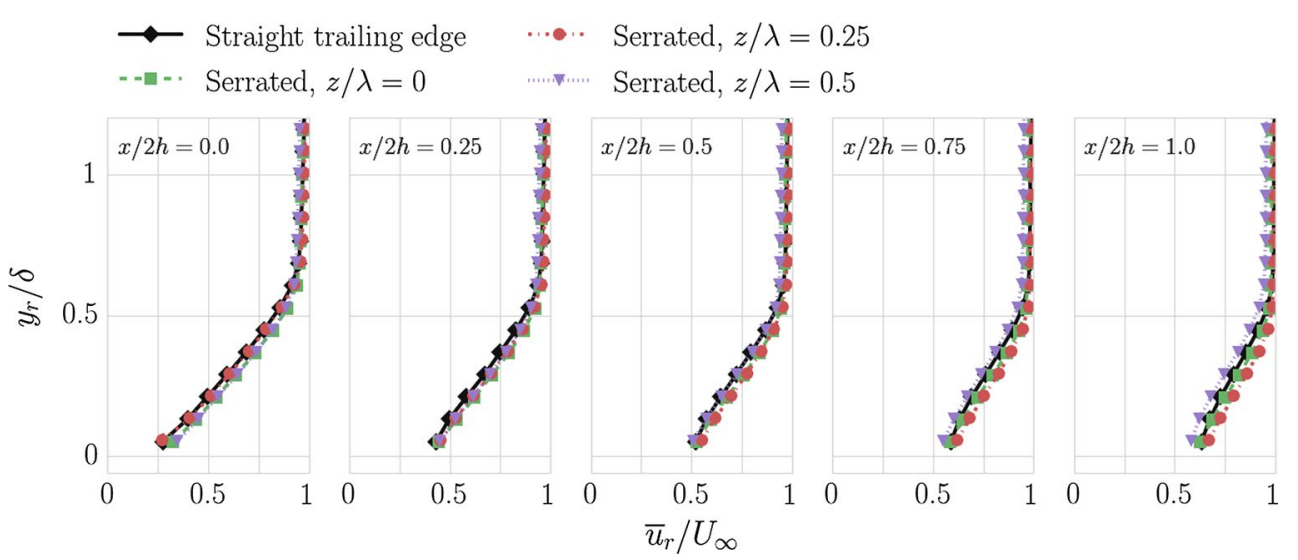

Fig. 13 Profiles over the wallnormal coordinate direction of $\overline{u_{r}}$ for straight and serrated trailing edge for $\alpha_{g}=12^{\circ}$ and $\varphi=0^{\circ}$. Suction side

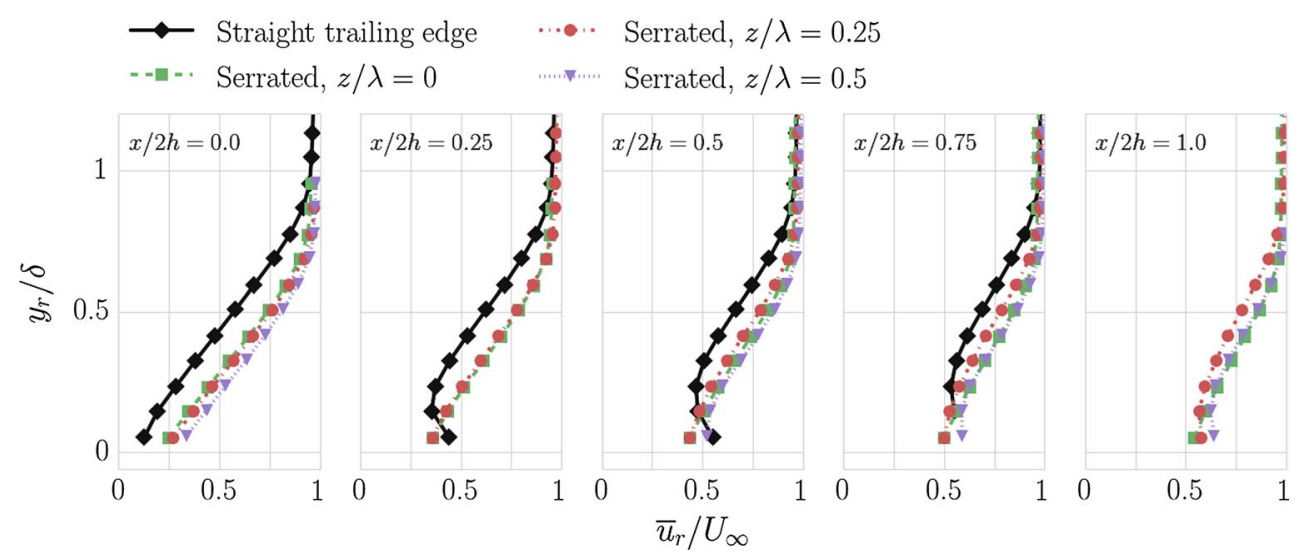

of streamlines, the result does not come to agree with the large differences (approximately $6 \mathrm{~dB}$ ) reported between the analytical solution of the serrated trailing edge noise, and the results current and previous of wind tunnel experiments (Gruber et al. 2013; Moreau and Doolan 2013).

Therefore, further elements are taken into consideration below, first of all the boundary layer thickness, shape factor, and the turbulent fluctuations.

\subsection{Boundary layer across serrations}

The properties of the flow across the serrations are inspected in three planes spanning one serration element.
These measurements were taken with the open test section setup, and as discussed above, the correction of the presented geometric angle of attack must be considered. The wall-normal distance, $y_{r}$, is normalized with the boundary layer thickness, $\delta$, measured at the straight trailing edge at $\alpha=0^{\circ}$ (see Sect. 2).

When the airfoil is at zero incidence and with zero flap angle, no significant difference is found between the straight edge and the cases with serrations (Fig. 12). At higher angles of attack, namely $\alpha_{g}=12^{\circ}\left(\alpha \approx 6.6^{\circ}\right)$, as shown in Fig. 13, clear differences emerge between the straight and serrated trailing edges. The former shows flow with a lower velocity region in the wake location, which 
Fig. 14 Time-averaged streamlines and contours of streamwise velocity component, $\bar{u}$. Straight trailing edge on the upper left, and varying spanwise positions of the serrated edge $\left(\varphi=0^{\circ}\right)$
Fig. 15 Wall-normal profiles of $u_{\mathrm{RMS}}^{\prime} / U_{\infty}$ for the straight and serrated trailing edges at different spanwise locations, $\alpha_{g}=0^{\circ}, \varphi=0^{\circ}$
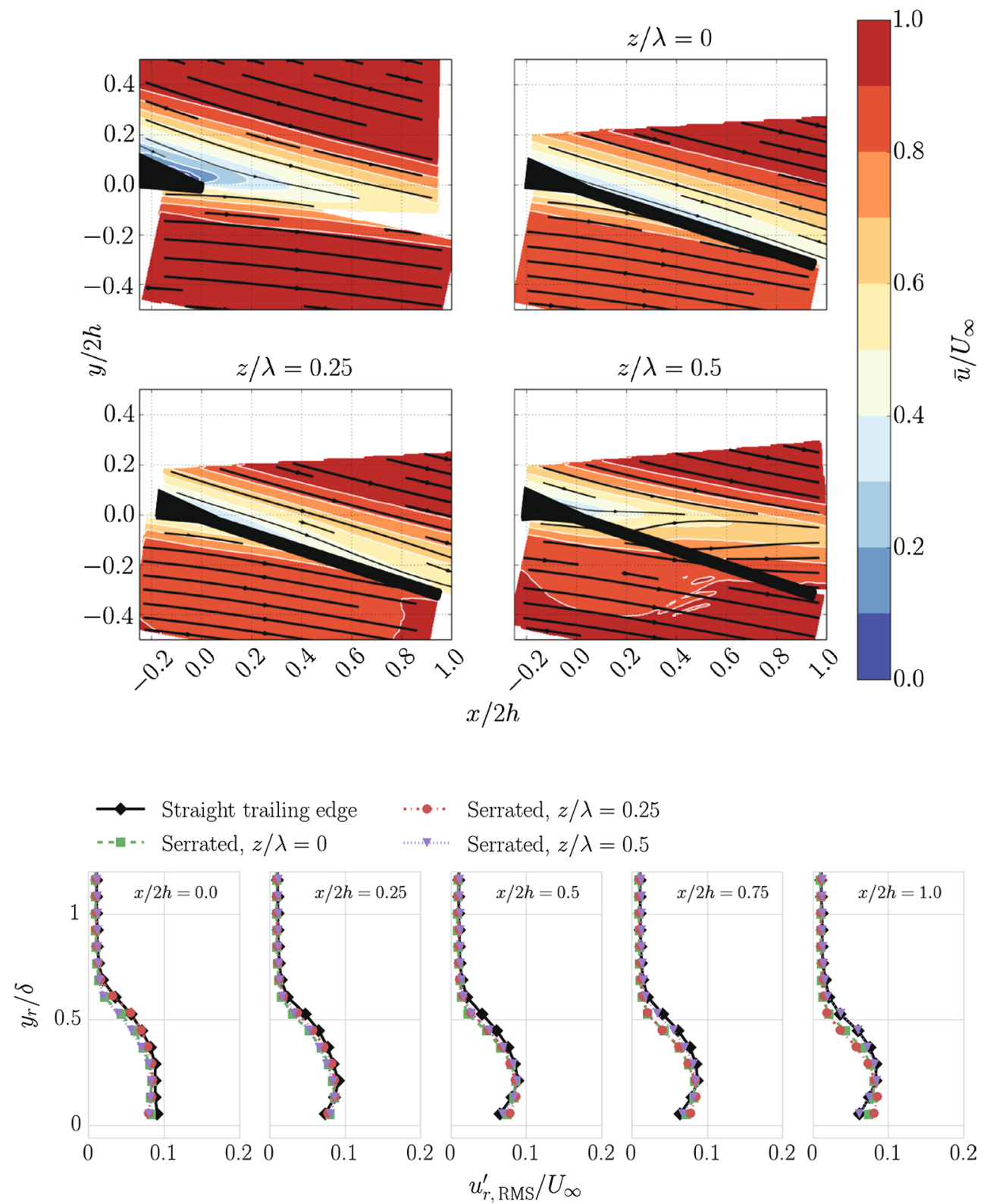

is replicated by the $z / \lambda=0.5$ location of the serrated edge after $x / 2 h=0.5$, and slightly by the $z / \lambda=0.25$ spanwise location further downstream.

The time-averaged streamwise velocity field is presented in Fig. 14. The value of $\alpha_{g}$ is $12^{\circ}$, and the serrations are placed at $\varphi=6^{\circ}$. The three spanwise measurement locations are presented for the serrated case. Contours of $\bar{u} / U_{\infty}$ are shown along with the projection of streamlines onto the examined planes.

Regions of lower velocity are observed close to the serration for the locations $z / \lambda=0$ and 0.5 . These two planes also exhibit a similar shape in the flow topology close to the serration surface and in its near wake. The flow in the measurement plane between adjacent serrations, $z / \lambda=0.5$, shows notable differences. Here the flow passing through adjacent serration teeth is evident, as shown previously in Fig. 8, as well as its influence on the shape, location, and thickness of the wake region.

\subsection{Turbulence statistics}

The mean flow topology near the serrations has been presented in the previous section and related to the discussion on serrated trailing edge noise in Howe (1991a, b). A look into the flow behavior from a turbulence statistics point of view will be given here.

The wall-normal profiles of $u_{\mathrm{RMS}}^{\prime}$ are given in Fig. 15 . No large differences are evident between the straight or serrated edges, nor for spanwise variations in the latter. The straight and the serrated edges at $z / \lambda=0.5$ do exhibit 
Fig. 16 Wall-normal values of $-\overline{u^{\prime} v^{\prime}} / U_{\infty}^{2}$ for the straight and serrated trailing edges at different spanwise locations, $\alpha_{g}=0^{\circ}, \varphi=0^{\circ}$
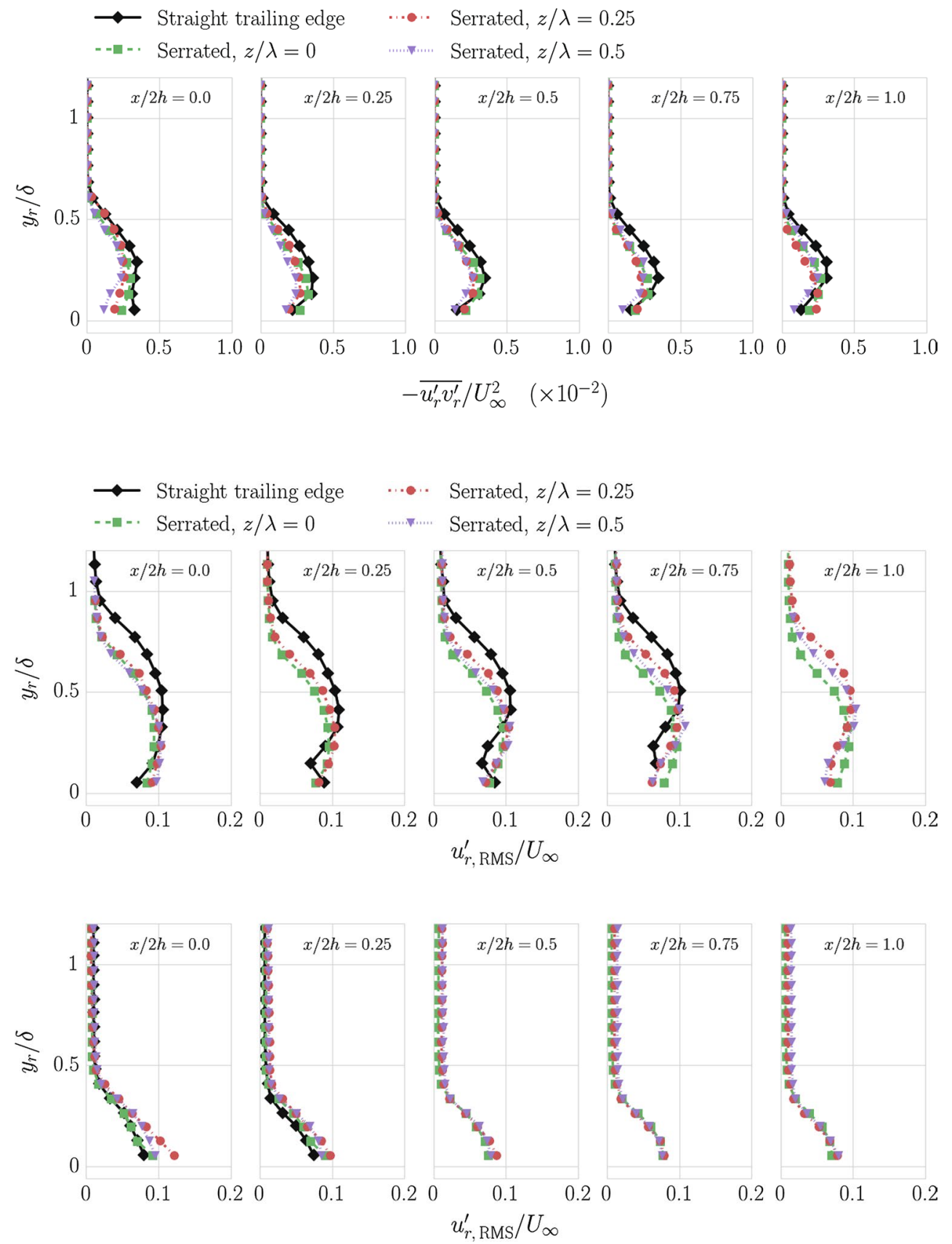

Fig. 17 Values of $u_{\mathrm{RMS}}^{\prime} / U_{\infty}$ for the straight and serrated trailing edges on the suction side (top) and pressure side (bottom), for different spanwise locations, $\alpha_{g}=12^{\circ}, \varphi=0^{\circ}$

similar behavior, with higher fluctuations seen further from $y / \delta=0$, and lower values closer to it. These similarities become more evident further downstream. The measured Reynolds stress, $-\overline{u^{\prime} v^{\prime}}$, shown in Fig. 16 show a similar trend, with overall little significant differences between the cases, but with the straight edge departing slightly from the serrated edge, which shows almost no spanwise variation.

Measurements of $u_{\mathrm{RMS}}^{\prime}$ for an airfoil incidence of $12^{\circ}$ (Fig. 17) show notable differences between straight and serrated trailing edges, and also along different spanwise locations of the latter. When $z / \lambda=0$, a behavior similar to what was seen for $\alpha_{g}=0^{\circ}$ for all streamwise locations is experienced. Measurements at $z / \lambda=0.25$ and 0.5 show a distinctly stronger decrease in fluctuation intensity around the wake centerline, an effect which is more apparent for locations further downstream. Values for the off-center spanwise locations are higher than for the $\alpha_{g}=0^{\circ}$ case for regions downstream of $x / 2 h=0.25$, but remain similar for the $z / \lambda=0$ location.

Measurements of $v_{\mathrm{RMS}}^{\prime}$ for different values of $\alpha_{g}$ and $\varphi$ are taken for $z / \lambda=0.25$ and shown in Fig. 18. At the suction side, cases at incidence or with flap deflection show higher values of $v_{\mathrm{RMS}}^{\prime}$ than for $\alpha_{g}=0^{\circ}, \varphi=0^{\circ}$. At $x / 2 h=0.5$, the serration trailing edge intersects the 
Fig. 18 Values of $v_{\mathrm{RMS}}^{\prime}$, suction side (top) and pressure side (bottom), for different values of $\alpha_{g}$ and $\varphi$ for the serrated trailing edge
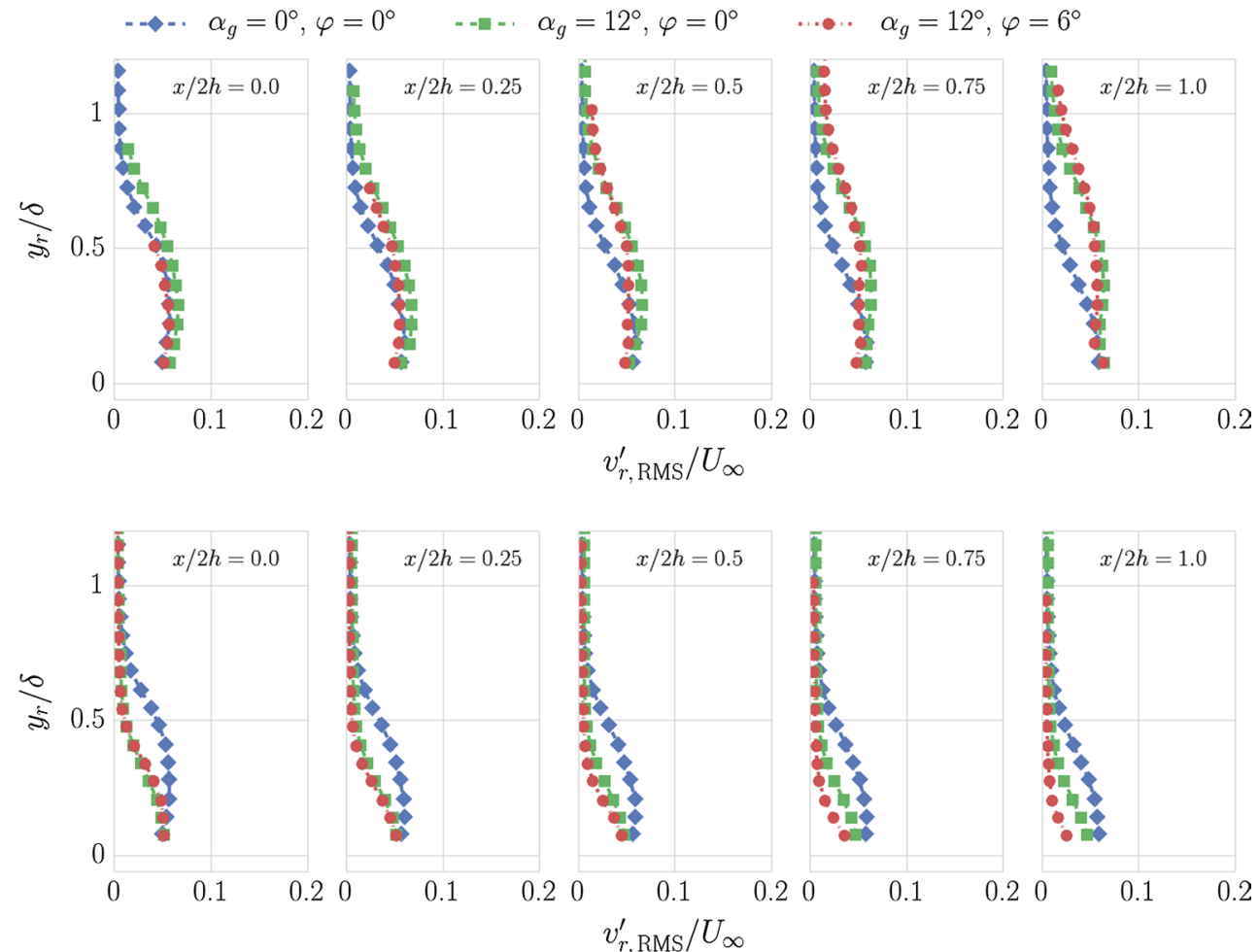

$v_{r, \text { RMS }}^{\prime} / U_{\infty}$

The Reynolds stress $-\overline{u_{r}^{\prime} v_{r}^{\prime}}$ of Fig. 19 shows overall higher values than for the zero incidence measurements. The $z / \lambda=0.5$ case approaches the profile of the straight edge for downstream locations, and $z / \lambda=0.25$ follows with less intensity but a similar shape. The serration centerline plane measurements remain distinctly different and follow the shape seen earlier for $\alpha_{g}=0^{\circ}$, but with higher values.

The spatial distribution of $-\overline{u_{r}^{\prime} v_{r}^{\prime}}$ is illustrated in Fig. 20 at the spanwise location of $z / \lambda=0.25$, where the measurement plane intersects the serration edge at $x / 2 h=0.5$. Values of $-\overline{u_{r}^{\prime} v_{r}^{\prime}}$ over the suction side attain a minimum for $\alpha_{g}=0^{\circ}, \varphi=0^{\circ}$. Instead, the cases with airfoil incidence and serration flap angle exhibit Reynolds shear stresses approximately three times higher, with the case $\alpha_{g}=12^{\circ}, \varphi=6^{\circ}$ showing the highest. The location of the maxima varies as well between cases, where for $\alpha_{g}=0^{\circ}, \varphi=0^{\circ}$ it is approximately at $y_{r} / \delta=0.25$, and for the other cases it increases from around $y_{r} / \delta=0.25$ at $x / 2 h=0$ to $y_{r} / \delta=0.7$ and 0.5 for $x / 2 h=1$. At the pressure side, the situation is similar to that observed for the wall-normal fluctuations, with the case $\alpha_{g}=0^{\circ}, \varphi=0^{\circ}$ yielding the highest values of $-\overline{u_{r}^{\prime} v_{r}^{\prime}}$, and also extending over a larger extent of $y_{r}$ before dropping to zero.

The amplitude of Reynolds shear stresses is associated to the importance of turbulent activity at relatively small scales in the boundary layer (see Ganapathisubramani $\alpha_{g}=0^{\circ}, \varphi=0^{\circ}$. This result is recalled in the discussion of Sect. 3.4. 
Fig. 19 Values of $-\overline{u_{r}^{\prime} v_{r}^{\prime}}$ for the straight and serrated trailing edges on the suction side (top) and pressure side (bottom) at different spanwise locations, $\alpha_{g}=12^{\circ}, \varphi=0^{\circ}$
Fig. 20 Values of $-\overline{u_{r}^{\prime} v_{r}^{\prime}}$, suction side (top) and pressure side (bottom) at different values of $\alpha_{g}$ and $\varphi$ for the serrated trailing edge
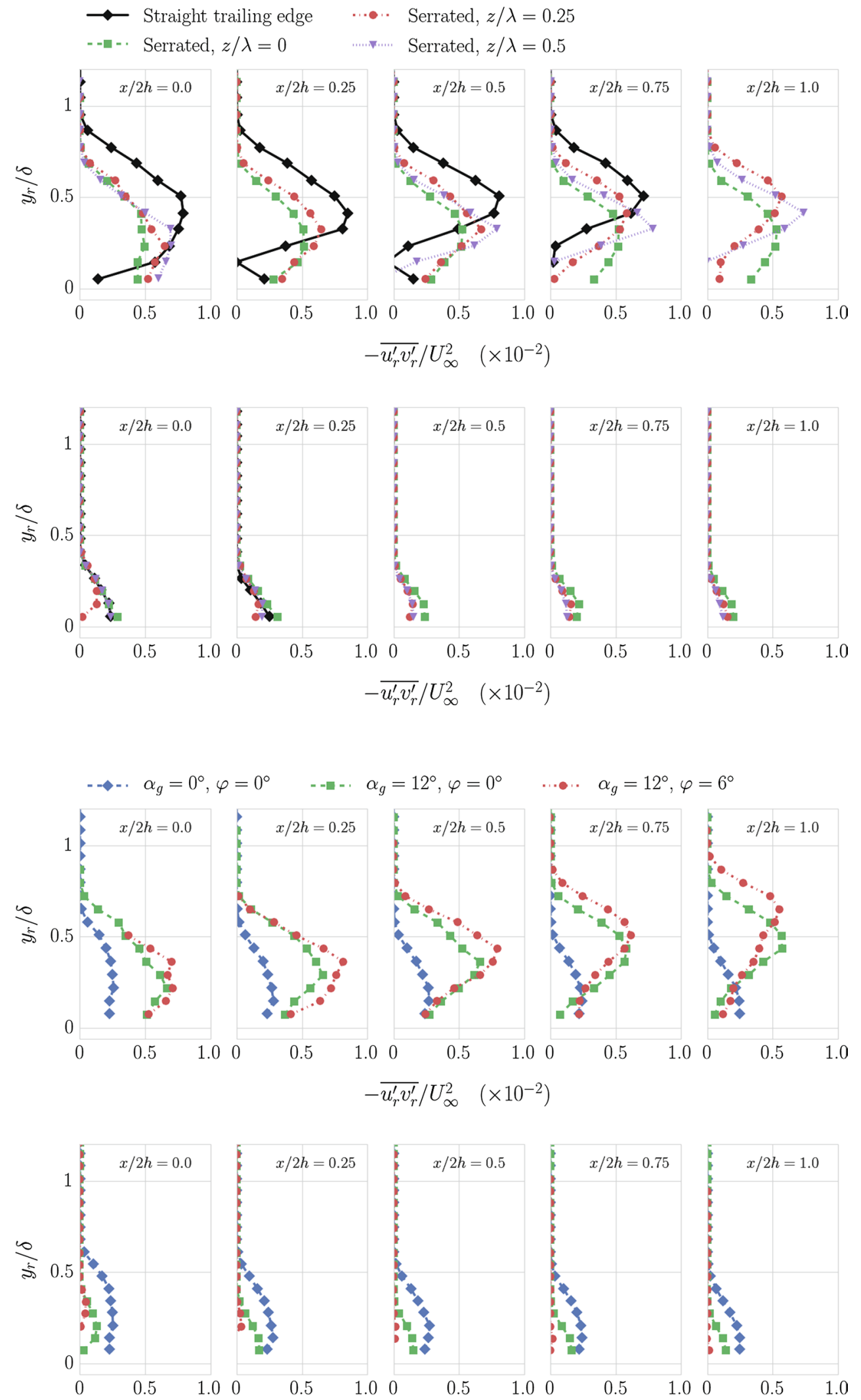

$-\overline{u_{r}^{\prime} v_{r}^{\prime}} / U_{\infty}^{2} \quad\left(\times 10^{-2}\right)$ 
Fig. 21 Velocity fluctuations of components $u_{\mathrm{RMS}}^{\prime}($ top $)$ and $v_{\mathrm{RMS}}^{\prime}($ bottom), for the straight edge (left), and the serrated edge $\left(\varphi=0^{\circ}\right.$ and $\left.6^{\circ}\right)$ at the spanwise location $z / \lambda=0.5$ and $\alpha_{g}=12^{\circ}$
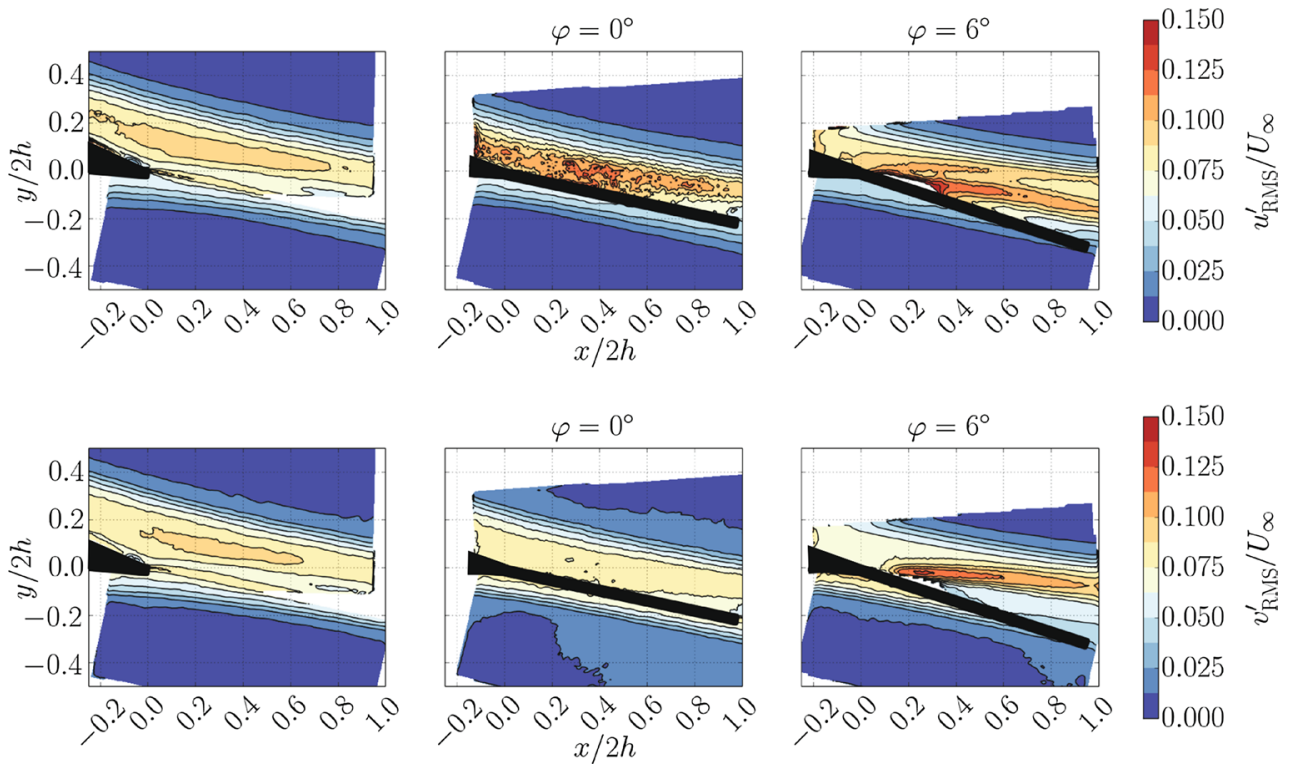

(Figs. 22 and 23). Higher levels of turbulence intensity can be seen for the $u$ component than for the $v$ component, supporting the observations made in the wall-normal plane. The $u$ component exhibits periodic and alternating up/down facing horseshoe-shaped regions of increased fluctuations behind the tips ( $\cup$ shaped), and the roots of the serrations ( $\cap$ shaped), with slightly higher $u_{\mathrm{RMS}}^{\prime}$ from the tip regions. The fluctuation intensity of the $v$ component (Fig. 23) shows similar patterns but is instead dominant in the region behind the serration root. This region is also where opposing streamwise vortices are in close proximity (see Fig. 7).

The $\alpha_{g}=0^{\circ}$ and $\varphi=0^{\circ}$ case shows no significant modulation along the spanwise direction. Increasing $\varphi$ causes a rapid increment of velocity fluctuations, which indicates that serrations at incidence cause an increase of turbulent fluctuations, in addition to altering the time-averaged streamlines patterns. Despite the above conditions, in the next section it is shown that the overall effect of serrations remains that of reducing the noise emissions (near $7 \mathrm{~dB}$ for some frequencies).

Observations in the cross-flow plane downstream of the serrations enable to visualize the spanwise distribution of the Reynolds shear stresses (Fig. 24). The airfoil incidence and the flap deflection introduce a significant increase of $-\overline{u_{r}^{\prime} v_{r}^{\prime}}$ with respect to the $\alpha_{g}=0^{\circ}, \varphi=0^{\circ}$ case. Positive maxima are formed trailing the serration teeth center, whereas negative-valued maxima emanate from the serration valleys. The value of these maxima is two to three times higher than that measured at zero incidence, with a slightly higher peak value originating from the pressure side shear layer. The overall pattern of Reynolds shear stresses is highly correlated with that of the vorticity distribution in Fig. 7, indicating an increase of turbulent kinetic energy production at airfoil incidence and flap deflection. cross-flow plane measurements at the wake of the serrations 
Fig. 22 Velocity fluctuations of the $u$ component in the crossflow plane
Fig. 23 Velocity fluctuations of the $v$ component in the crossflow plane

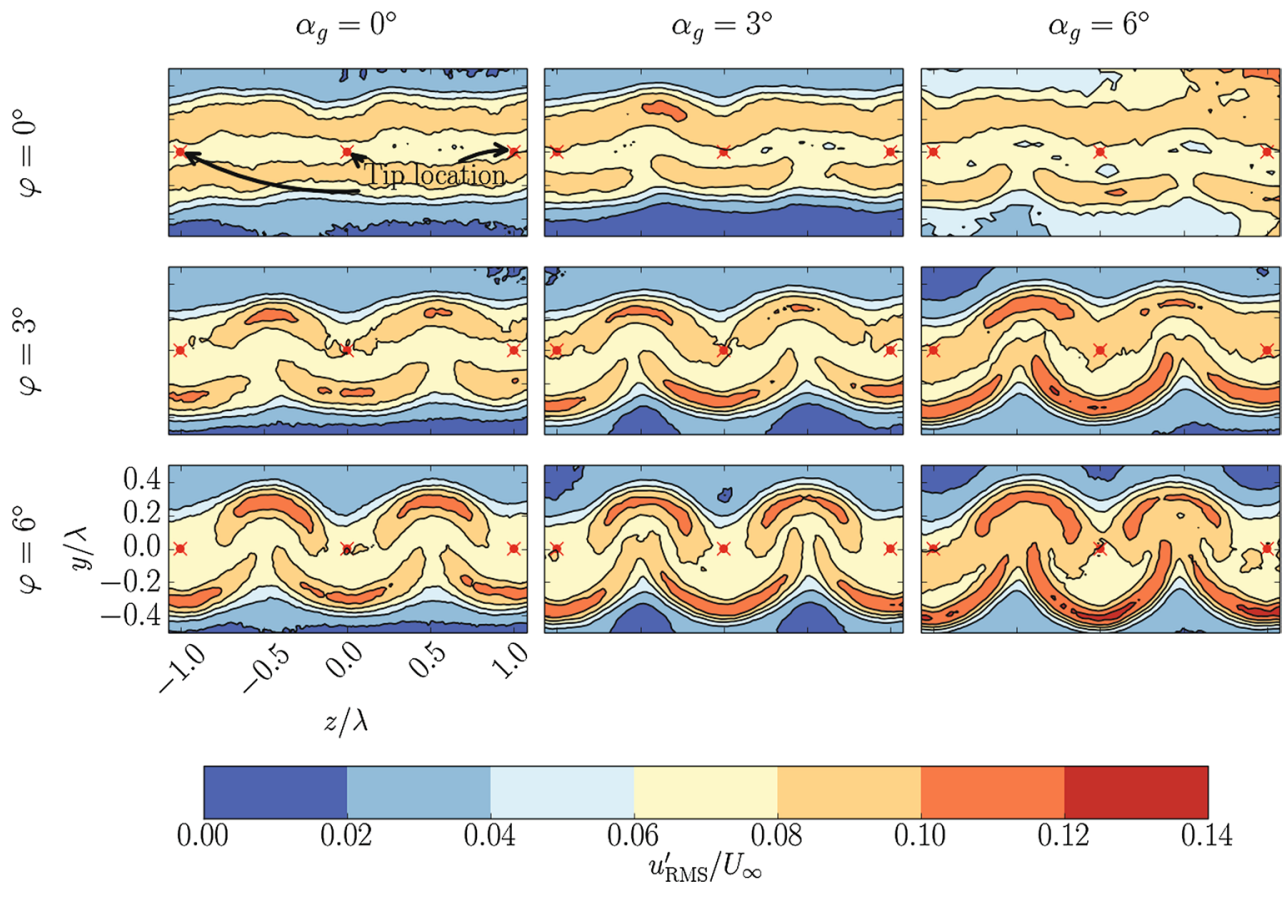

$\alpha_{g}=0^{\circ}$

$\alpha_{g}=3^{\circ}$

$\alpha_{g}=6^{\circ}$

$$
8
$$
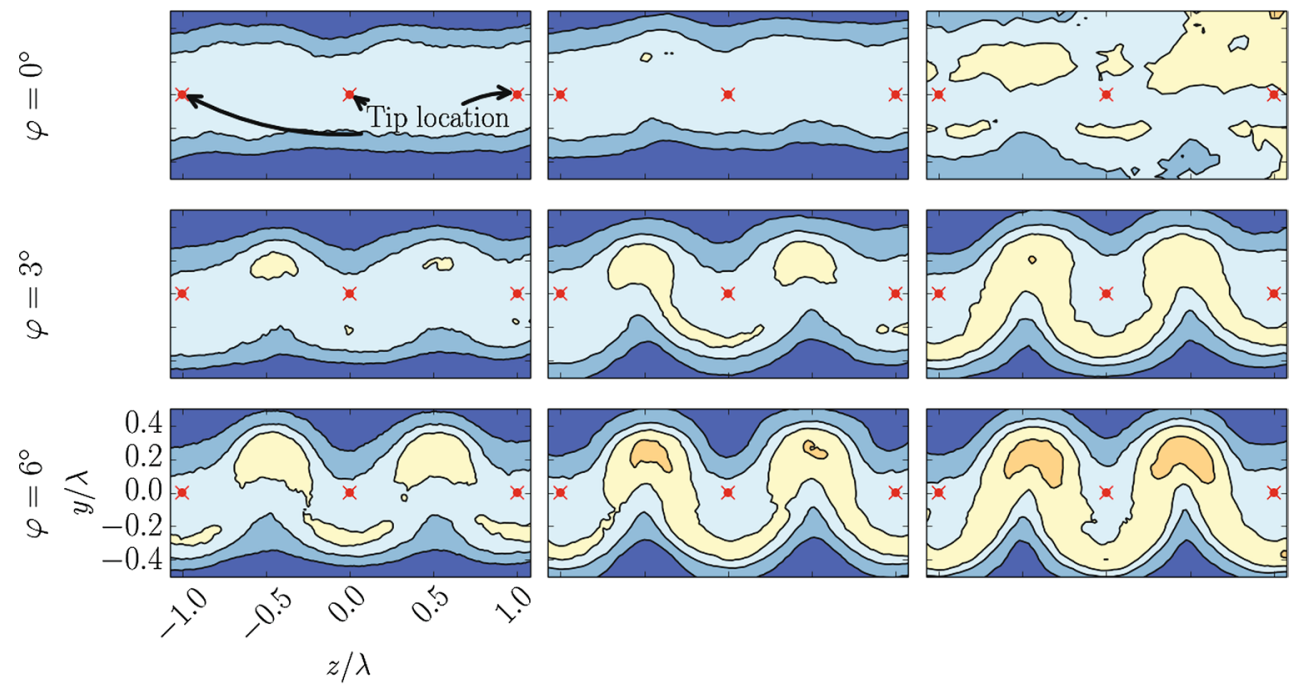

The overall conclusion is that the Reynolds shear stress increase is more pronounced than that of $u^{\prime}$ (approximately $30-50 \%)$ and $v^{\prime}(50-70 \%)$, which preludes to an increase of sound production especially at the high-frequency side of the spectrum. In the following section, evidence is gathered on the above increase (also referred to as loss of noise reduction efficiency) of serrations when placed at incidence.

\subsection{Noise emissions}

The third-octave band sound pressure level, SPL, of the straight trailing edge airfoil and the serrated airfoil can be seen in Fig. 25 for $\alpha_{g}=0^{\circ}$ and $U_{\infty}=35 \mathrm{~m} / \mathrm{s}$. The serrations are shown to be effective at reducing the noise emitted by the airfoil trailing edge. Also, there is a marked 
Fig. 24 Values of the Reynolds stress $-\overline{u_{r}^{\prime} v_{r}^{\prime}}$ in the cross-flow plane

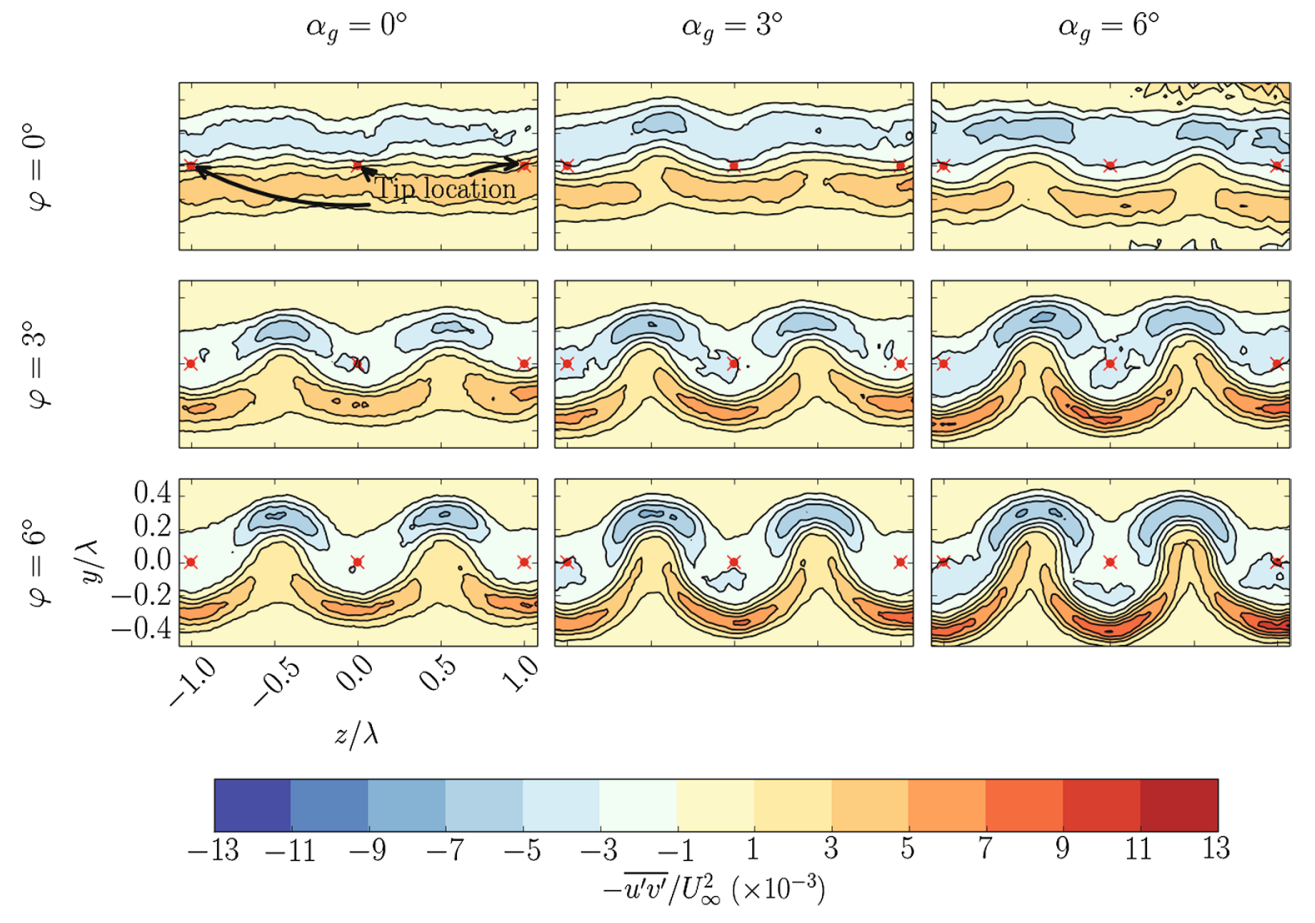

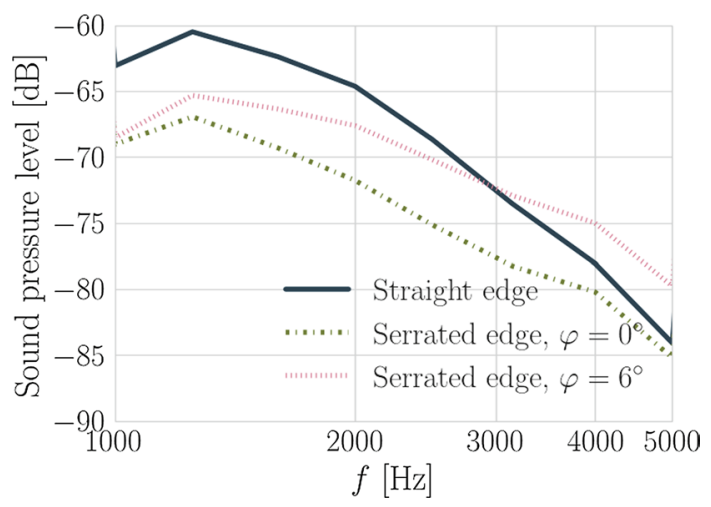

Fig. 25 Third-octave sound pressure levels for the straight, and two serrated trailing edges, with $\alpha_{g}=0^{\circ}$ and $U_{\infty}=35 \mathrm{~m} / \mathrm{s}$

difference between the noise measured using the serrations with $\varphi=0^{\circ}$ and those with $\varphi=6^{\circ}$.

Results of the measured noise for the serrations relative to the straight edge are shown in Fig. 26. The three airfoil incidence cases and the two serration flap angles are presented for a freestream velocity of $35 \mathrm{~m} / \mathrm{s}$. The vertical axis represents the measured sound pressure level difference in decibels with respect to the straight trailing edge, where a positive number indicates a reduction in noise and a negative value represents an increase in noise.

At $\varphi=0^{\circ}$ (Fig. 26, top), the serrations offer a reduction of up to $7 \mathrm{~dB}$ for the frequency band between $1 \mathrm{kHz}$ and $3 \mathrm{kHz}$. Beyond this upper limit, the reduction vanishes when approaching $5 \mathrm{kHz}$. At $\varphi=0^{\circ}$ the change in angle of attack has only a small effect on the noise reduction.

When the serrations are placed at $\varphi=6^{\circ}$ (Fig. 26, bottom), the noise reduction is less pronounced, to the point that, depending on airfoil incidence, an increase of noise is measured in the higher frequency range. This increase may relate to the presence of high RMS in the flow caused by these high flap or airfoil incidence angles, as discussed in the previous section. The frequency where such inversion occurs is denoted as crossover frequency, $f_{0}$, and has been reported in detail in Gruber et al. (2010).

The approximation of Howe (1991b) for the noise of a similar serration geometry on the trailing edge of an infinitely thin flat plate under the same flow conditions, without incidence, is also presented in Fig. 26 (solid line). The differences observed with the experimentally measured noise from the serration-retrofitted airfoil are notable. A variation regarding the spectrum shape is persistent, where the present case shows higher reduction at the lower and mid-frequency range presented, and less reduction in the higher frequencies. Instead, the prediction indicates an opposite behavior. This contrast has also been reported in Gruber et al. (2011). Alternatively, a simplistic application of Eq. (4) based on the spectrum of the straight edge in Fig. 26 would be approximated as a constant reduction for all frequencies, thus represented as a straight line, at the same predicted constant reduction discussed above in Fig. 11, indicating an overestimation of at least $5 \mathrm{~dB}$ over the reduction levels here observed. Its inclusion is omitted for conciseness. 

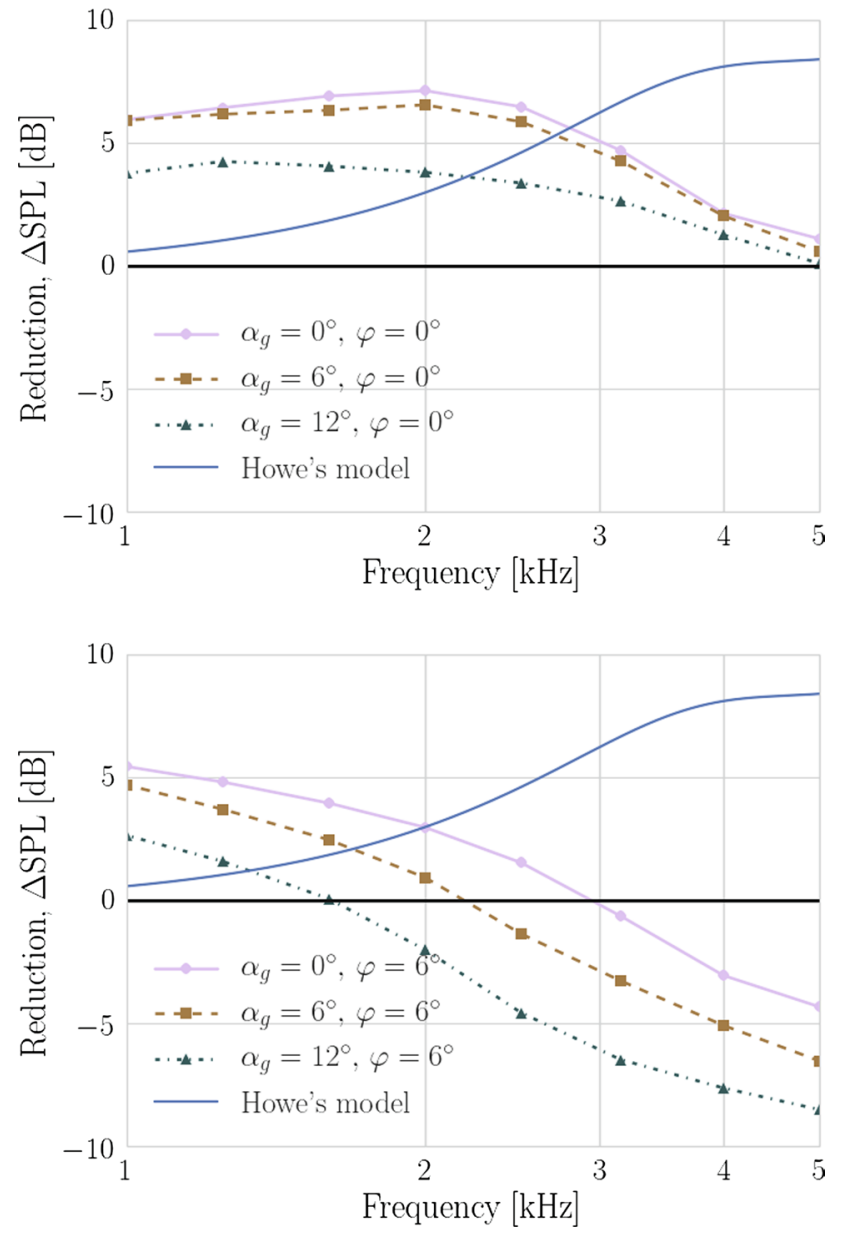

Fig. 26 Measured noise differences in third-octave band SPL of serrations relative to the straight trailing edge for $U_{\infty}=35 \mathrm{~m} / \mathrm{s}$, for $\varphi=0^{\circ}$ (top) and $6^{\circ}$ (bottom). The noise of the serrated trailing edge of a flat plate according to Howe (1991b) is also presented, for which only the $\alpha_{g}=0^{\circ}$ and $\varphi=0^{\circ}$ case is applicable

Although the authors have determined that the degree of serration-flow misalignment significantly affects the efficiency at which the serrations reduce noise, the fact that the discrepancies between the predicted and measured levels of reduction are present for the $\alpha_{g}=0^{\circ}, \varphi=0^{\circ}$ case, indicates that misalignment by itself cannot be used to explain them. A comprehensive study of the boundary layer flow properties, especially using time-resolved information, could help identify further reasons for this discrepancy. This analysis demands a dedicated scrutiny and is reserved for an independent paper.

\section{Conclusions}

The mean topology and the turbulence statistics of the flow near trailing edge serrations have been studied using PIV under different degrees of airfoil incidence and serration flap angle. The PIV measurements yield the pattern of streamlines close to the surface of serrations enabling to quantify their departure from the freestream direction. Measurements in the cross-flow plane indicate the presence of streamwise vortices emanating from the serrated edge, with their strength primarily influenced by the serration flap angle. The results of the mean flow measurements are input to a simplified version of the model in Howe (1991b) that estimates relative noise reduction on the basis of the local angle between the flow and the trailing edge.

The study is complimented with acoustic measurements, by which it is shown that the serrated trailing edge effectively reduces the turbulent boundary layer trailing edge noise of the airfoil, although to a lesser extent than that which the prediction suggests. This is consistent with experimental findings reported in the literature. The PIV survey further reveals that, in the $\alpha_{g}=0^{\circ}, \varphi=0^{\circ}$ case, the mean flow and turbulence statistics do not exhibit significant changes, yet a noticeable noise reduction is achieved. When serration-flow misalignment is prescribed, the flow undergoes important changes in various mean flow and turbulence statistic measures. Despite this significant departure from the assumed conditions in the Howe model, the noise is still reduced in the lower frequencies of the range measured. At higher frequencies, the noise is instead increased. Changes in turbulence statistics between serration-flow misaligned cases and the $\alpha_{g}=0^{\circ}, \varphi=0^{\circ}$ case serve to explain the observed change in acoustic emissions.

Acknowledgments The research of Carlos Arce is funded by the Innovation Fund Denmark, Industrial Ph.D. Programme Project Number 11-109522. The authors would further like to thank Andreas Fischer (Danish Technical University) for the fruitful conversations.

Open Access This article is distributed under the terms of the Creative Commons Attribution 4.0 International License (http://creativecommons.org/licenses/by/4.0/), which permits unrestricted use, distribution, and reproduction in any medium, provided you give appropriate credit to the original author(s) and the source, provide a link to the Creative Commons license, and indicate if changes were made.

\section{References}

Arce C, Ragni D, Pröbsting S, Scarano F (2015) Flow field around a serrated trailing edge at incidence. In: 33rd Wind Energy Symposium, American Institute of Aeronautics and Astronautics, Kissimmee, Florida. doi:10.2514/6.2015-0991

Arina R, Della Ratta Rinaldi R, Iob A, Torzo D (2012) Numerical study of self-noise produced by an airfoil with trailing-edge serrations. In: 18th AIAA/CEAS Aeroacoustics Conference (33rd AIAA Aeroacoustics Conference), AIAA, Colorado Springs, Colorado, USA, June, pp 4-6. doi:10.2514/6.2012-2184

Bertagnolio F, Fischer A, Jun Zhu W (2014) Tuning of turbulent boundary layer anisotropy for improved surface pressure and trailing-edge noise modeling. J Sound Vib 333(3):991-1010. doi:10.1016/j.jsv.2013.10.008 
Blake WK (2012) Mechanics of flow-induced sound and vibration V2: complex flow-structure interactions, vol 2. Elsevier, Amsterdam

Brooks T, Marcolini M, Pope D (1984) Airfoil trailing edge flow measurements and comparison with theory, incorporating open wind tunnel corrections. In: 9th Aeroacoustics Conference, AIAA, Williamsburg, Virigina. doi:10.2514/6.1984-2266

Brooks T, Pope D, Marcolini M (1989) Airfoil self-noise and prediction. NASA Reference Publication Number 1218

Brooks TF, Hodgson TH (1981) Trailing edge noise prediction from measured surface pressures. J Sound Vib 78(1):69-117

Bull MK (1979) On the form of the wall-pressure spectrum in a turbulent boundary layer in relation to noise generation by boundary layer-surface interactions. In: Mechanics of sound generation in flows; Proceedings of the Joint Symposium, pp 210-216

Chong TP, Vathylakis A (2015) On the aeroacoustic and flow structures developed on a flat plate with a serrated sawtooth trailing edge. J Sound Vib. doi:10.1016/j.jsv.2015.05.019

Dassen T, Parchen R, Bruggeman J, Hagg F (1996) Results of a wind tunnel study on the reduction of airfoil self-noise by the application of serrated blade trailing edges. Tech. rep, National Aerospace Laboratory, NLR

Finez A, Jondeau E, Roger M, Jacob MC (2011) Broadband noise reduction of a linear cascade with trailing edge serrations. In: 17th AIAA/CEAS Aeroacoustics Conference (32nd AIAA Aeroacoustics Conference), Portland, Oregon, USA. doi:10.2514/6.2011-2874

Ganapathisubramani B, Longmire EK, Marusic I (2003) Characteristics of vortex packets in turbulent boundary layers. J Fluid Mech 478:35-46. doi:10.1017/S0022112002003270

Ghaemi S, Scarano F (2013) Turbulent structure of high-amplitude pressure peaks within the turbulent boundary layer. J Fluid Mech 735:381-426. doi:10.1017/jfm.2013.501

Gruber M (2012) Airfoil noise reduction by edge treatments. Ph.D. thesis, University of Southampton

Gruber M, Azarpeyvand M, Joseph PF (2010) Airfoil trailing edge noise reduction by the introduction of sawtooth and slitted trailing edge geometries. In: Proceedings of 20th International Congress on Acoustics, ICA 10(August):1-9

Gruber M, Joseph P, Chong T (2011) On the mechanisms of serrated airfoil trailing edge noise reduction. In: 17th AIAA/CEAS Aeroacoustics Conference (32nd AIAA Aeroacoustics Conference), American Institute of Aeronautics and Astronautics, Portland, Oregon, USA, vol 2781, pp 5-8. doi:10.2514/6.2011-2781

Gruber M, Joseph P, Azarpeyvand M (2013) An experimental investigation of novel trailing edge geometries on airfoil trailing edge noise reduction. In: 19th AIAA/CEAS Aeroacoustics Conference, AIAA, Berlin, Germany. doi:10.2514/6.2013-2011
Howe M, Street C (1999) Trailing edge noise at low Mach numbers. J Sound Vib 225(2):211-238. doi:10.1006/jsvi.1999.2236

Howe MS (1991a) Aerodynamic noise of a serrated trailing edge. J Fluids Struct 5(1):33-45. doi:10.1016/0889-9746(91)80010-B

Howe MS (1991b) Noise produced by a sawtooth trailing edge. J Acoust Soc Am 90(1):482. doi:10.1121/1.401273

Moreau D, Doolan C (2013) Noise-reduction mechanism of a flat-plate serrated trailing edge. AIAA J 51(13):2513-2522. doi:10.2514/1.J052436

Moreau D, Brooks L, Doolan C (2011) Flat plate self-noise reduction at low-to-moderate Reynolds number with trailing edge serrations. In: Proceedings of the Annual Conference on the Australian Acoustical Society

Mueller T (2002) Aeroacoustic measurements. Springer Science \& Business Media, Berlin

Oerlemans S, Fisher M, Maeder T, Kögler K (2009) Reduction of wind turbine noise using optimized airfoils and trailing-edge serrations. AIAA J 47(6):1470-1481. doi:10.2514/1.38888

Pröbsting S, Tuinstra M, Scarano F (2015a) Trailing edge noise estimation by tomographic particle image velocimetry. J Sound Vib 346:117-138. doi:10.1016/j.jsv.2015.02.018

Pröbsting S, Zamponi M, Ronconi S, Guan Y, Morris S, Scarano F (2016) Vortex shedding noise from a beveled trailing edge. Int J Aeroacoust (in press)

Raffel M, Willert C, Kompenhans J (2007) Particle image velocimetry. Experimental fluid mechanics. Springer, Berlin. doi:10.1007/978-3-540-72308-0

Schepers J, Curvers A, Oerlemans S, Braun K, Lutz T, Herrig A, Wuerz W, Mantesanz A, Fischer M, Koegler K, Maeder T, Garcillán L, Others, Méndez-López B, Matesanz A, Ahrelt R (2007) Sirocco: silent rotors by acoustic optimisation. In: Second International Meeting on Wind Turbine Noise

Schrijer FFJ, Scarano F (2008) Effect of predictor-corrector filtering on the stability and spatial resolution of iterative PIV interrogation. Exp Fluids 45(5):927-941. doi:10.1007/s00348-008-0511-7

Sijtsma P (2010) Phased array beamforming applied to wind tunnel and fly-over tests. Tech. rep., SAE Technical Paper. doi:10.4271/2010-36-0514

Stern F, Muste M, Beninati M, Eichinger W (1999) A study of experimental uncertainty assessment methodology with example. Tech. rep., Iowa Institute of Hydraulic Research, College of Engineering, The University of Iowa, Iowa City, USA

Westerweel J (1997) Fundamentals of digital particle image velocimetry. Meas Sci Technol 8(12):1379-1392. doi:10.1088/0957-0233/8/12/002

Wieneke B (2015) PIV uncertainty quantification from correlation statistics. Meas Sci Technol. doi:10.1088/0957-0233/26/7/074002 